\title{
THE CLASSIFICATION PROBLEM FOR MODELS OF ZFC
}

\author{
by \\ Samuel Dworetzky
}

\author{
A thesis \\ submitted in partial fulfillment \\ of the requirements for the degree of \\ Master of Science in Mathematics \\ Boise State University
}

May 2017 
(C) 2017

Samuel Dworetzky

ALL RIGHTS RESERVED 
BOISE STATE UNIVERSITY GRADUATE COLLEGE

DEFENSE COMMITTEE AND FINAL READING APPROVALS

of the thesis submitted by

Samuel Dworetzky

Thesis Title: The Classification Problem for Models of ZFC

Date of Final Oral Examination: 07 March 2017

The following individuals read and discussed the thesis submitted by student Samuel Dworetzky, and they evaluated the presentation and response to questions during the final oral examination. They found that the student passed the final oral examination.

Samuel Coskey Ph.D. Chair, Supervisory Committee

John Clemens, Ph.D. Member, Supervisory Committee

Marion Scheepers, Ph.D. Member, Supervisory Committee

The final reading approval of the thesis was granted by Samuel Coskey Ph.D., Chair of the Supervisory Committee. The thesis was approved by the Graduate College. 


\begin{abstract}
Models of ZFC are ubiquitous in modern day set theoretic research. There are many different constructions that produce countable models of ZFC via techniques such as forcing, ultraproducts, and compactness. The models that these techniques produce have many different characteristics; thus it is natural to ask whether or not models of ZFC are classifiable. We will answer this question by showing that models of ZFC are unclassifiable and have maximal complexity. The notions of complexity used in this thesis will be phrased in the language of Borel complexity theory.

In particular, we will show that the class of countable models of ZFC is Borel complete. Most of the models in the construction as it turns out are ill-founded. Thus, we also investigate the sub problem of identifying the complexity for well-founded models. We give partial results for the well-founded case by identifying lower bounds on the complexity for these models in the Borel complexity hierarchy.
\end{abstract}




\section{Contents}

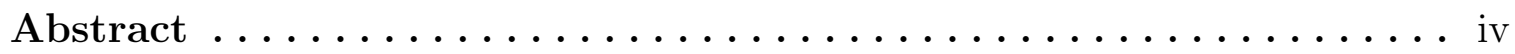

1 Introduction......................... 1

1.1 Models of LO, PA, and ZFC $\ldots \ldots \ldots \ldots \ldots \ldots \ldots \ldots \ldots \ldots$

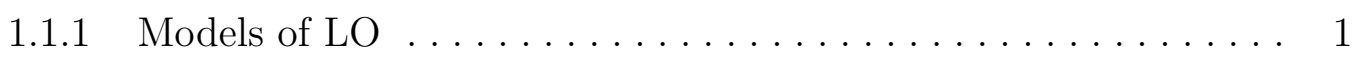

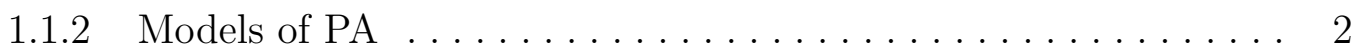

1.1.3 Models of ZFC $\ldots \ldots \ldots \ldots \ldots \ldots \ldots \ldots \ldots \ldots$

1.2 Borel Classification Theory....................... 4

1.3 Summary of results.............................. 6

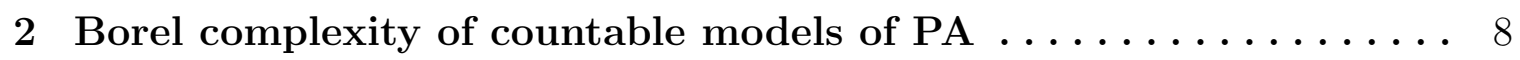

2.1 Ehrenfeucht-Mostowski Models $\ldots \ldots \ldots \ldots \ldots \ldots \ldots \ldots \ldots$

2.2 Building canonical extensions for models of PA $\ldots \ldots \ldots \ldots \ldots 11$

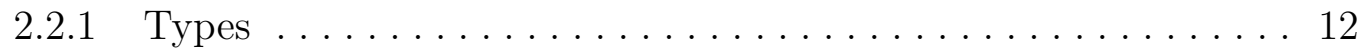

2.2.2 End extensions of models of PA generated by types ....... 13

2.3 Extensions of Models of PA along linear orders . . . . . . . . . . . 19

2.4 Borel reducibility of linear orders to models of PA . . . . . . . . . . 21

3 Borel complexity of countable models of ZFC . . . . . . . . . 24

3.1 Types with parameters from $w \ldots \ldots \ldots \ldots \ldots \ldots \ldots \ldots \ldots$

3.2 End extensions of models of ZFGC generated by types $\ldots \ldots \ldots \ldots 27$

3.3 Canonical extensions of models of ZFGC along linear orders . . . . . . 30 


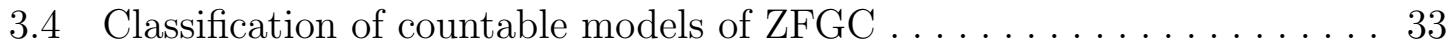

4 The classification of Well-Founded Models of ZFC . . . . . . . . 36

4.1 Generating $\omega_{1}$ many non-isomorphic well-founded models of a comple-

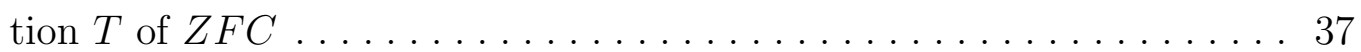

4.2 Generating $2^{\omega}$ many non-isomorphic well-founded models of $Z F C \ldots 38$

4.3 Generating $E_{0}$ many non-isomorphic well-founded models of a comple-

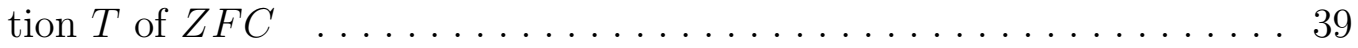

Bibliography $\ldots \ldots \ldots \ldots \ldots \ldots \ldots \ldots \ldots \ldots \ldots \ldots \ldots \ldots \ldots \ldots \ldots \ldots$ 


\section{Chapter 1}

\section{INTRODUCTION}

Mathematical structures exist in many varieties. Thus, it is natural to ask, given a particular class of structures, whether or not we can provide a means of classification. Borel complexity theory is an active area that provides a framework for classifying mathematical structures. Using this framework, we are able to gauge the complexity of mathematical structures. In particular, we will focus on the complexity of models of Peano arithmetic and set theory.

The classification problem for models of Peano arithmetic was addressed in a paper

of Coskey and Kossak [1]. In this paper, they showed that models of Peano arithmetic have maximal complexity. The result is proved by establishing that models of Peano arithmetic are just as complex as the class of countable linear orders which have maximal complexity, which was addressed by Friedman and Stanley in the article [3]. We will make the notion of maximal complexity precise in section 1.2.

\subsection{Models of LO, PA, and ZFC}

\subsubsection{Models of LO}

In Chapter 2 we will relate countable models of PA to models of countable linear orders. Here we will formally define the axioms for a linear ordering. 
Definition 1.1.1. Let $I$ be a set, and $\leq$ be a binary relation. Then, $I^{\prime}=(I, \leq)$ is a linear order, denoted $I^{\prime} \models L O$ with language $\mathcal{L}_{L O}=\{\leq\}$ if the following axioms are satisfied:

(1) $\forall a, b \in I(a \leq b \wedge b \leq a \Rightarrow a=b)$

(Antisymmetry)

(2) $\forall a, b, c \in I(a \leq b \wedge b \leq c \Rightarrow a \leq c)$

(Transitivity)

(3) $\forall a, b \in I(a \leq b \vee b \leq a)$

(Comparability)

Example 1.1.1. Let $N=(\mathbb{N}, \leq)$ where $\leq$ is interpreted in the usual way. It is clear that $N \models L O$, using properties of $\mathbb{N}$.

\subsubsection{Models of PA}

In this section, we will state precisely what we mean by a model of Peano arithmetic. Informally, a model of Peano arithmetic or PA is a structure in which all of classical number theory can be carried out. Formally, a model of PA is defined as follows:

Definition 1.1.2. A countable model $M$ of $P A$, denoted $M \models P A$, is a first order structure with underlying domain $\mathbb{N}$ and language $\mathcal{L}_{P A}=\{+, \times,<, 0,1\}$ satisfying the following axioms:

(1) $\forall x \forall y \forall z((x+y)+z=x+(y+z))$

(2) $\forall x \forall y(x+y=y+x)$

(3) $\forall x \forall y \forall z((x \times y) \times z=x \times(y \times z)$

(4) $\forall x \forall y(x \times y=y \times x)$ 
(5) $\forall x \forall y \forall z(x \times(y+z)=x \times y+x \times z)$

(6) $\forall x((x+0=x) \wedge(x \times 0=0))$

(7) $\forall x(x \times 1=x)$

(8) $\forall x \forall y \forall z((x<y \wedge y<z) \rightarrow x<z)$

(9) $\forall x(x \nless x)$

(10) $\forall x \forall y(x<y \vee x=y \vee y<x)$

(11) $\forall x \forall y \forall z(x<y \rightarrow x+z<y+z)$

(12) $\forall x \forall y \forall z(0<z \wedge x<y \rightarrow x \times z<y \times z)$

(13) $\forall x \forall y(x<y \rightarrow \exists z(x+z=y))$

(14) $0<1 \wedge \forall x(0<x \rightarrow 1 \leq x)$

(15) $\forall x(0 \leq x)$

(16) If $\varphi$ is a formula in the language $\mathcal{L}_{P A}$ then,

$\forall \bar{y}(\varphi(0, \bar{y}) \wedge \forall x(\varphi(x, \bar{y} \rightarrow \varphi(x+1, \bar{y})) \rightarrow \forall x \varphi(x, \bar{y}))$

We note that the standard model of PA is the structure $(\mathbb{N},+, \times,<, 0,1)$ in which each symbol is interpreted with its usual meaning, and satisfies the axioms of Definition 1.1.2. On the other hand, there are so called nonstandard models of PA which are those structures $M \models P A$ that are not isomorphic to $\mathbb{N}$. Nonstandard models of PA where first exhibited by Skolem in the 1930's using the compactness theorem of first order logic. For reference we also note that $M \models P A^{-}$simply means that $M$ is a model of axioms (1) - (15) only.

\subsubsection{Models of ZFC}

We now provide a formal definition of a model of set theory which we will also refer to as a model of ZFC. 
Definition 1.1.3. A model $M$ of $Z F C$, denoted $M=Z F C$ is a first order structure with language $\mathcal{L}_{Z F C}=\{E\}$ where $E$ is a binary relation symbol satisfying the following axioms:

(1) Extensionality: $\forall x \forall y(\forall z(z E x \leftrightarrow z E y) \rightarrow x=y)$

(2) Foundation: $\forall x(\exists y(y E x) \rightarrow \exists y(y E x \wedge \neg \exists z(z E x \wedge z E y))$

(3) Comprehension: For each formula $\varphi$ in the language $\mathcal{L}_{Z F C}$ with free variables $x, z, \bar{w}$

$\forall z \forall \bar{w} \exists y \forall x(x E y \leftrightarrow x E z \wedge \varphi(x, z, \bar{w}))$

(4) Pairing: $\forall x \forall y \exists z(x E z \wedge y E z)$

(5) Union: $\forall u \exists y \forall s \forall x(x E s \wedge s E u \rightarrow x E y)$

(6) Replacement: For each formula $\varphi$ in the language $\mathcal{L}_{Z F C}$ with free variables $x, y, z, \bar{w}$, $\forall z \forall \bar{w}(\forall x(x E z \exists ! y(\varphi(x, y, z, \bar{w}) \rightarrow \exists v \forall x(x E z) \exists y(y E v) \varphi(x, y, z, \bar{w})))$

(7) Infinity: $\exists x(\emptyset E x \wedge \forall y(y E x)(S(y) E x))$

(8) Power set: $\forall x \exists y \forall z(\forall w(w E z \rightarrow w E x) \rightarrow z E y)$

(9) Choice: $\forall x \exists y$ y well orders $x$.

As with models of PA, there are both standard and non standard models of ZFC. A standard model of ZFC is one in which the E relation from Definition 1.1.3 is the actual $\in$ set membership relation. Furthermore, by the Mostowski Collapse lemma we know that every well-founded model is isomorphic to a standard model of set theory. Thus we refer to standard models sometimes as well-founded models.

\subsection{Borel Classification Theory}

In this section, we will introduce some aspects of Borel Classification theory that allow us to assess the complexity of the classification of countable models of PA and 
ZFC. To study the complexity of a countable class of structures satisfying a complete theory $T$, we first look at the space $X_{T}=\{M: \operatorname{dom}(M)=\omega \wedge M \models T\}$. By a complete theory we mean a theory that has a model, and any two models of this theory are elementarily equivalent. The classification problem for models $M \models T$ is determined by studying the isomorphism equivalence relation $\cong_{T}$ on the space $X_{T}$. We note that if $a(R)$ is the arity of logical symbol in the language $\mathcal{L}_{T}$ then we can view $X_{T}$ as a Borel subset of the space $\prod_{R \in \mathcal{L}} 2^{\mathbb{N}^{a(R)}}$ of all countable $\mathcal{L}$-structures.

In order to discuss the complexity of the isomorphism relation $\cong_{T}$, we need the notion of Borel reducibility. Thus, we provide the following definition.

Definition 1.2.1. Let $X$ and $Y$ be standard Borel spaces, and $E$ and $F$ be equivalence relations on $X$ and $Y$ respectively. Then, $E$ is Borel Reducible to $F$, denoted $E \leq_{B} F$ if there is a Borel function $f: X \rightarrow Y$ such that $x E y \leftrightarrow f(x) F f(y)$.

Relating this definition to our specific context, this means that if $E$ is Borel reducible to $F$ then the classification problem for structures in the space $Y$ up to $F$ is at least as complex as the classification problem for structures in the space $X$ up to $E$. Later we will show that the isomorphism relation for certain classes of structures is maximally complex among countable structures, or Borel complete. Formally, we have the following definition:

Definition 1.2.2. The class of countable models of a theory $T$ is Borel Complete if and only if for any other class of countable models of a theory $T^{\prime}$ there is a Borel reduction $\cong_{T^{\prime}} \leq_{B} \cong_{T}$. 
Example 1.2.1. The class $\left(X_{L O}, \cong_{L O}\right)$ of countable linear orders under isomorphism is Borel complete [3].

In general there are many other notions of complexity that are "less" complex than Borel complete. Other complexities that will arise in this thesis are $=, E_{0}$, and $E_{\omega_{1}}$.

Definition 1.2.3. The identity relation on a space $X$ which we denote $i d(X)$ is defined as $i d(X)=\{(x, y) \in X \times X: x=y\}$.

In particular, we will examine the identity relation on $2^{\omega}$ in Chapter 4 . We next define the relation of eventual agreement on $2^{\omega}$.

Definition 1.2.4. Let $x, y \in 2^{\omega}$. Then, the relation $E_{0}$ is defined as follows, $x E_{0} y \Leftrightarrow$ $\exists m \forall n \geq m x(n)=y(n)$.

We finally define the $E_{\omega_{1}}$ equivalence relation. This relation is designated for classes of structures with $\omega_{1}$ many equivalence classes.

Definition 1.2.5. Consider the space $X_{W O} \subset X_{L O}$ of countable well ordered structures. Then, $E_{\omega_{1}}$ is the isomorphism relation on the space $X_{W O}$.

We note that that the space $X_{W O}$ is not Borel, and thus does not technically exists in the Borel equivalence relation hierarchy. This limitation will not effect our results in Chapter 4.

\subsection{Summary of results}

As stated in the introduction, models of PA are known to be Borel complete. In Chapter 2, we will examine the argument used in [1] showing that models of PA are Borel complete. The idea is that we can find a Borel reduction from the isomorphism 
relation on the class of countable linear orders to the isomorphism relation on the class of countable models of PA. In particular, the chapter focuses on constructing a model of PA from a linear order in such a way that we can identify the order type of the original linear order.

In Chapter 3, we show that the class of countable models of ZFC is Borel complete. Again, we show that the isomorphism relation on the class of countable linear orders is Borel reducible to the isomorphism relation on countable models of ZFC. The argument we use in Chapter 3 is similar to the construction outlined in Chapter 2. We adapt this construction by adjoining witnesses of a minimal type with parameters from the $\omega$ of our fixed model of ZFC.

Note that the models we produce in Chapter 3 are generally ill-founded. It is then natural to ask whether we can find complexity within just the well-founded models of ZFC. In Chapter 4, we provide three constructions of such models. We first construct $\omega_{1}$ many models using the inner model $L$. Then, we generate $2^{\omega}$ many using mutual Cohen generics, and finally $E_{0}$ many by adapting the construction for $2^{\omega}$ many. 


\section{Chapter 2}

\section{BOREL COMPLEXITY OF COUNTABLE MODELS OF PA}

This chapter will establish the following result due to Coskey-Kossak: the class of countable models of PA is Borel complete [1]. We know that the class of countable linear orders is Borel complete. Thus, it suffices to show that the isomorphism relation for countable linear orders is Borel reducible to the isomorphism relation on countable models of PA. The presentation of the material on Ehrenfeucht-Mostowski models was borrowed from [6]. The presentation for constructing the models $M(I)$ was borrowed from [7], [8], [5], and [9].

\subsection{Ehrenfeucht-Mostowski Models}

In this section we will briefly discuss Ehrenfeucht-Mostowski models. The results in this section will be applied in the last section of this chapter to show that models of PA are Borel complete.

We begin by defining order indiscernibles. For convenience, we will fix a language $\mathcal{L}$ and an $\mathcal{L}$-structure $\mathcal{M}$ with domain $M$; and refer to this structure in the following definitions and theorems in the section. 
Definition 2.1.1. Consider the ordered set $(I,<)$, and a sequence $\left\langle a_{i}: i \in I\right\rangle$ such that each $a_{i} \in M$. The sequence $\left\langle a_{i}: i \in I\right\rangle$ is a sequence of order indiscernibles if $\mathcal{M} \models \varphi\left(a_{i_{1}}, \ldots, a_{i_{n}}\right) \leftrightarrow \varphi\left(a_{j_{1}}, \ldots, a_{j_{n}}\right)$ whenever $i_{1}<\ldots<i_{n}$ and $j_{1}<\ldots<j_{n}$ are elements of the index set $I$.

Let $T$ be an $\mathcal{L}$ theory of our fixed language. The following theorems require our theory to contain built in Skolem functions. Using theorems from elementary model theory, we can always extend our theory $T^{*} \supseteq T$ and language $\mathcal{L}^{*} \supseteq \mathcal{L}$ that contains built in Skolem functions.

Definition 2.1.2. Let $\mathcal{M} \models \mathcal{L}^{*}$, and consider a subset $X \subseteq M$. Then, we define the smallest elementary $\mathcal{L}^{*}$-substructure generated by $X$ to be the Skolem hull. We denote this substructure by $\mathcal{H}(X)$.

Definition 2.1.3. An Ehrenfeucht-Mostowski model is any Skolem hull of a sequence of order indiscernibles of any model $\mathcal{M}=T^{*}$. We denote this model by $\mathcal{H}(I)$ where $I$ is a set of order indiscernibles.

We finally introduce the notion of the type of a sequence of order indiscernibles.

Definition 2.1.4. Consider a sequence of order indiscernibles $X=\left\langle a_{i}: i \in I\right\rangle$ of the model $\mathcal{M}$. Then, the type of the sequences of indiscernibles is defined as $\operatorname{tp}(X)=\left\{\psi\left(x_{1}, \ldots, x_{n}\right): \mathcal{M} \models \psi\left(a_{i_{1}}, \ldots, a_{i_{n}}\right)\right.$ whenever $\left.i_{1}<\ldots<i_{n} \in I\right\}$

The following lemma shows us that if our theory has built in Skolem functions, then isomorphisms of linear orderings can be extended to isomorphisms of EurenfeuchtMostowski models. We will apply this result in a later section to models of PA. This 
is possible since models of PA have built in Skolem functions.

Lemma 2.1.1. Let $T^{*}$ be an $\mathcal{L}^{*}$-theory with built in Skolem functions. Let I be a sequence of order indiscernibles in a model $\mathcal{M} \models T^{*}$ and $J$ be sequence of order indiscernibles in a model $\mathcal{N}=T^{*}$ such that $t p(I)=t p(J)$. Then, if $f: I \rightarrow J$ is an isomorphism, we can extend $f$ to an isomorphism $g: \mathcal{H}(I) \rightarrow \mathcal{H}(J)$.

Proof. Let $a \in \mathcal{H}(I)$ and $a_{1}, \ldots, a_{n} \in I$ such that $a=t\left(a_{1}, \ldots, a_{n}\right)$ for a Skolem term $t$. Define $g(a)=t\left(f\left(a_{1}\right), \ldots, f\left(a_{n}\right)\right)$. We claim that the map $g$ is well defined and an isomorphism.

To show that $g$ is well defined, consider a Skolem term $s \neq t$ where $a=s\left(a_{1}, \ldots, a_{n}\right)=$ $t\left(a_{1}, \ldots, a_{n}\right)$. So, $\mathcal{M} \models t\left(a_{1}, \ldots, a_{n}\right)=s\left(a_{1}, \ldots, a_{n}\right)$. Since $\operatorname{tp}(I)=\operatorname{tp}(J)$ and $f$ is an isomorphism, it follows that $\mathcal{N} \models t\left(f\left(a_{1}\right), \ldots, f\left(a_{n}\right)\right)=s\left(f\left(a_{1}\right), \ldots, f\left(a_{n}\right)\right)$. Hence, $g\left(t\left(a_{1}, \ldots, a_{n}\right)\right)=g\left(s\left(a_{1}, \ldots, a_{n}\right)\right)$ and thus $g$ is well defined.

Now we will show that $g$ is an elementary embedding. We claim that $g$ is injective. Let $a=t\left(a_{1}, \ldots, a_{n}\right)$ and $b=t^{\prime}\left(a_{1}, \ldots, a_{n}\right)$. Suppose $g(a)=g(b)$. Since $g(a)=g(b)$, it follows that $\mathcal{N} \models t\left(f\left(a_{1}\right), \ldots, f\left(a_{n}\right)\right)=t^{\prime}\left(f\left(a_{1}\right), \ldots, f\left(a_{n}\right)\right)$. Since $t p(I)=t p(J)$, it follows that $\mathcal{M}=a=t\left(a_{1}, \ldots, a_{n}\right)=t^{\prime}\left(a_{1}, \ldots, a_{n}\right)=b$. Hence, $g$ is injective.

Now, we will show that $g$ preserves relation symbols. Fix $R \in \mathcal{L}$ such that $R$ is a relation symbol and consider $b_{1}, \ldots, b_{n} \in \mathcal{H}(I)$. Let $b_{i}=t_{k}\left(b_{i, 1}, \ldots, b_{i, n}\right)$ such that $k \in\{1, \ldots, n\}$ and $b_{i, j} \in I$ for each $i, j \in\{1, . ., n\}$. We need to show that $\left(b_{1}, \ldots, b_{n}\right) \in R^{\mathcal{H}(I)} \Leftrightarrow\left(g\left(b_{1}\right), \ldots, g\left(b_{n}\right) \in R^{\mathcal{H}(J)}\right.$. Observe the following chain of logical equivalences 


$$
\begin{aligned}
\left(g\left(b_{1}\right), \ldots, g\left(b_{n}\right)\right) \in R^{\mathcal{H}(J)} & \Leftrightarrow\left(t_{1}\left(f\left(b_{1,1}\right), . ., f\left(b_{1, n}\right)\right), . ., t_{n}\left(f\left(b_{n, 1}\right), . ., f\left(b_{n, n}\right)\right)\right) \in R^{\mathcal{H}(J)} \\
& \Leftrightarrow\left(t_{1}\left(b_{1,1}, . ., b_{1, n}\right), . ., t_{n}\left(b_{n, 1}, . ., b_{n, n}\right)\right) \in R^{\mathcal{H}(I)} \\
& \Leftrightarrow\left(b_{1}, . ., b_{n}\right) \in R^{\mathcal{H}(I)} .
\end{aligned}
$$

Hence, $g$ preserves relation symbols.

Now we will show that $g$ preserves function symbols. Let $h$ be a function symbol in our fixed language, and $b_{1}, \ldots, b_{n} \in \mathcal{H}(I)$. We know that $M \models h^{\mathcal{H}(I)}\left(b_{1}, \ldots, b_{n}\right)=$ $c$ for some $c \in \mathcal{H}(I)$. Since $g$ preserves relation symbols, it follows that $N \models$ $h^{\mathcal{H}(J)}\left(g\left(b_{1}, \ldots, b_{n}\right)\right)=g(c)=g\left(h^{\mathcal{H}(I)}\left(b_{1}, \ldots, b_{n}\right)\right)$. Thus, $g$ preserves function symbols.

We will now show that $g$ maps constant symbols in $\mathcal{H}(I)$ to constants in $\mathcal{H}(J)$. Let $c \in \mathcal{L}^{*}$ be a constant symbol. We can write $c=t\left(a_{1}, \ldots, a_{n}\right)$ where each $a_{i} \in I$ for all $i \in\{1, \ldots, n\}$. Then, by definition of $g, g\left(c^{\mathcal{H}(I)}\right)=t\left(f\left(a_{1}, \ldots, f\left(a_{n}\right)\right)\right)$. Since $t\left(f\left(a_{1}, \ldots, f\left(a_{n}\right)\right)\right)$ is a constant in $\mathcal{H}(J)$, it follows that $g$ maps constant symbols in $\mathcal{H}(I)$ to constant symbols in $\mathcal{H}(J)$.

Finally, we show that $g$ is surjective. Fix $b \in \mathcal{H}(J)$. We can write $b=t\left(b_{1}, \ldots, b_{n}\right)$ for some Skolem term $t$ such that $b_{1}, \ldots, b_{n} \in J$. Since $f$ is surjective we can find $a_{1}, \ldots, a_{n} \in I$ such that $f\left(a_{i}\right)=b_{i}$ for all $i \in\{1, \ldots, n\}$. Then, by definition of $g$ we have that $t\left(f\left(a_{1}\right), . ., f\left(a_{n}\right)=g\left(t\left(a_{1}, . ., a_{n}\right)\right)\right.$. Hence, $g$ is surjective. Thus, we have shown that $g$ is an isomorphism.

\subsection{Building canonical extensions for models of PA}

In this section we will discuss extensions of models of PA that are generated by unbounded strongly definable complete types. We begin this section by defining 
items concerning types.

\subsubsection{Types}

We will now discuss the notion of a type. A type is simply a consistent collection of formulas, that is a collection of formulas satisfied by some model. We will also define types with special properties that will be used to construct extensions of models of PA.

Definition 2.2.1. Let $T$ be an $\mathcal{L}$-theory and $\mathcal{M} \models T$. Then, we say an $\boldsymbol{n}$-type $p(\bar{v})$ is a collection of formulas in the language $\mathcal{L}$ with $n$-many free variables $\bar{v}$ such that parameters of $p$ come from $\mathcal{M}$.

We will only consider 1 -types for the rest of this paper, and refer to these simply as types $M$-types.

Remark 2.2.1. The set Def(M) will refer to the definable sets in $M$.

Definition 2.2.2. Let $p(v)$ be an $M-$ type. We say $p(v)$ is a complete type over $M$ if for all $\theta(v) \in \mathcal{L}(M)$ either $\theta(v) \in p(v)$ or $\neg \theta(v) \in p(v)$.

Definition 2.2.3. Let $M \models P A$. An $M$-type $p(v)$ over the model $\mathcal{M}$ is unbounded if $p(v) \supseteq\{v>x: x \in M\}$.

Definition 2.2.4. Let $M \models P A$. Then a complete $M$-type $p(v)$ is strongly definable if for every formula $\varphi(v, z) \in \mathcal{L}$, we can find a formula $\theta(v) \in p(v)$ with the following property,

$$
M \models \forall z\left(\forall^{\infty} v(\theta(v) \rightarrow \varphi(v, z)) \vee \forall^{\infty} v(\theta(v) \rightarrow \neg \varphi(v, z))\right)
$$


In other words, strongly definable types contain formulas that "eventually" settle the truth value of any formula in a given language.

Fact 2.2.1. In any model $M$ of Peano arithmetic, there is an unbounded strongly definable type [5].

Definition 2.2.5. Let $p(v)$ be a complete $M$-type. Then, $p(v)$ is definable if the set $\{z \in M: \forall \varphi(v, z) \in \mathcal{L} \varphi(v, z) \in p(v)\}$ is in $\operatorname{Def}(M)$.

\subsubsection{End extensions of models of PA generated by types}

Definition 2.2.6. Let $\mathcal{M} \models T$ where $T$ is a complete and consistent extension of $P A$. Then, the model $\mathcal{M}$ is prime if for any other model $\mathcal{N} \models T$ we can find an elementary embedding from $\mathcal{M}$ to $\mathcal{N}$.

Definition 2.2.7. Let $\mathcal{L}$ be a language and $\mathcal{M}$ an $\mathcal{L}$ structure. Now, we the expanded language $\mathcal{L}_{\mathcal{M}}$ by adding a new constant symbol for each element of the domain of $\mathcal{M}$. Thus, we say the elementarydiagram of $\mathcal{M}$, denoted Diagel $(\mathcal{M})$, is the set of $\mathcal{L}_{\mathcal{M}}$ sentences that are true in $\mathcal{M}$.

Definition 2.2.8. Let $M \models P A$ and $p(v)$ be a complete $M-$ type, then $M(p / d)$ is the prime model of $\operatorname{Diag}_{e l}(M) \cup p(d)$.

Remark 2.2.2. We will write $M(p)$ if a priori we are not referring to a specific element satisfying the type $p$.

Question 2.2.1. What do these extensions actually look like? 
We can characterize extensions of models of PA as the "ground" model $M \models P A$ in addition to a convex set that live within the new model. These convex sets are called gaps, and are generated by the new constant symbol $d$. We will later find that if we assume a minimal type, then the gaps will all lie above the original model.

Definition 2.2.9. Let $d \in M \models P A . M(d)=\{x \in M: x<t(d)$ for some Skolem function $t\}$.

In other words, $M(d)$ is the smallest elementary initial segment that contains $d$.

Definition 2.2.10. Let $d \in M \models P A . M[d]=\{x \in M: t(x)<d$ for every Skolem function $t\}$.

We can think of $M[d]$ as all possible ways of applying Skolem functions in the model that are still less than $d$. One can also think of this set as the largest elemtary initial segment excluding the element $d$.

We now arrive at the definition of gap by deleting elements that cannot reach $d$ via any Skolem function.

Definition 2.2.11. Let $d \in M \models P A$. Now, we define gap $(d)=M(d) \backslash M[d]$.

We can characterize gaps using the following fact. That is, a gap of a point $d$ is characterized by finding a Skolem function that "jumps" over $d$ from some other point $c$ in the gap, and vice versa. 
Fact 2.2.2. Let $c, d \in M \models P A . c \in g a p(d)$ if and only if there are Skolem functions $t, s$ such that $t(c) \geq d$ and $s(d) \geq c$.

Proof. Let $c \in \operatorname{gap}(d)$. Then, $c \in M(d)$ and $c \notin M[d]$. Since $c \in M(d), c<t(d)$ for some Skolem function $\mathrm{t}$, and $c \notin M[d]$ it follows that $d \leq s(c)$ for some Skolem function $s$.

Remark 2.2.3. One can easily show that the relation of being in the same gap is an equivalence relation. Thus, it follows that gaps in any model of PA are disjoint.

The next four lemmas we state will be used in the proof of Proposition 2.2.1.

Lemma 2.2.1. If $x<y \in M[d]$, then for every Skolem function $t, t(x) \leq \max \left\{t\left(y^{\prime}\right)\right.$ : $\left.y^{\prime} \leq y\right\}<d$.

Proof. Let $x<y \in M[d]$. Fix a Skolem function $t$. Since $x<y \in M[d]$, it follows that $t(y)<d$ and $t(x)<d$. Notice that $t(x) \leq \max \left\{t\left(y^{\prime}\right): y^{\prime} \leq y\right\}$. Consider any $y^{\prime} \leq y$. Since $y \in M[d], y^{\prime} \in M[d]$. Hence, $t\left(y^{\prime}\right)<d$. Thus, $t(x) \leq \max \left\{t\left(y^{\prime}\right): y^{\prime} \leq\right.$ $y\}<d$.

The next several lemmas show that definable types generate end extensions of models of PA. We prove this fact by first showing that definable types generate what are known as conservative types, and then show that conservative types are end extensional. 
Definition 2.2.12. We say that an extension $M \subseteq K$ of a model $M \models P A$ is conservative, if for any set $X$ definable with parameters from $K$, then $X \cap M \in$ $D E F(M)$.

Lemma 2.2.2. $M \models P A$, and $p(v)$ a complete $M$ - type. If $p$ is a definable type, then $M(p)$ is a conservative extension of $M$.

Proof. Let $d \in M(p)$ realizing the type $p$. We want to show that $M(p)$ is a conservative extension. Let $X \in \operatorname{Def}(M(p))$. Then, $X=\{\bar{z} \in M(p): M(p) \models \theta(c, z)\}$ such that $\theta$ is a formula in the language of arithmetic and $c \in M(p)$. Since $c \in M(p)$, we have that $x=\min (x) \eta(x, d)$ for some formula $\eta$ in the language of arithmetic. We must show that $X \cap M \in \operatorname{Def}(M(p))$.

Notice that $X \cap M=\{z \in M: M(p) \models \theta(\min (x) \eta(x, d), \bar{z})\}$ since $c=\min (x) \eta(x, d)$. So, we have that $\{z \in M: M(p) \models \theta(\min (x) \eta(x, d), z)\}=\{z \in M: \theta(\min (x) \eta(x, d), z) \in$ $p(v)\}$. Finally, since $p$ is a definable type it follows that $X \cap M \in \operatorname{Def}(M)$. Hence, $M(p)$ is a conservative extension.

Remark 2.2.4. The converse of Lemma 2.2.2 is also true [9].

Lemma 2.2.3. Conservative extensions $K \supseteq M \models P A$ with $K \models P A^{-}$are end extensional.

Proof. Let $K$ be a conservative extension of a model $M \models P A$ where $K \models P A^{-}$. Fix $a \in K \backslash M$. Consider the set $X=\{x \in K: x<a\}$. Note that the set $X$ is definable 
in $K$. Since $K$ is a conservative extension, it follows that $X \cap M=\{x \in M: x<$ $a\} \in \operatorname{Def}(M)$. The set $X \cap M$ is contains the "0" element. Furthermore, for any $y \in M$, the successor $s(y) \in M$. Thus, we conclude by induction that $X \cap M=M$ and $M<a$. Hence, $K$ is an end extension.

Lemma 2.2.4. If $M \models P A^{-}$then any unbounded strongly definable complete $M-$ type is definable.

The following proposition is due to Gaifman, and provides us with a way to visualize end extensions generated by unbounded strongly definable complete $M-$ types [7]. These extensions consist of the ground model of arithmetic, as well as the gap generated by some element $d$ over the given type. It should also be noted that the gap of $d$ is generated above the ground model.

Proposition 2.2.1. $M \models P A$, and $p(v)$ be an unbounded strongly definable complete $M$-type. Then, $M(p / d)$ is the disjoint union of $M$ and $g a p(d)$.

Proof. We know that $p(v)$ is unbounded. So, in particular $p(d) \supset\{d>x: x \in M\}$ and thus $d>M$. By Lemma 2.2.1 we know that $t(x)<d$ for any Skolem function $t$ and $x \in M$. We now claim that $M \subseteq M(p / d)[d]$ where $M(p / d)[d]=\{x \in M(p / d)$ : $t(x)<d$ for any Skolem function $\mathrm{t}\}$.

Let $x \in M$. Then $x \in M(p / d)$. Since $t(x)<d$ for any Skolem function $\mathrm{t}$ and $x \in M$, it follows that $x \in M(p / d)[d]$. Hence, $M \subseteq M(p / d)[d]$.

Now we claim that $M \cap g a p(d)=\emptyset$. If not, then we can find $x \in M \cap g a p(d)$. Note that $x \in M(p / d)[d]$ implies that $t(x)<d$ for every $t$. On the other hand, $x \in g a p(d)$ implies that $d \leq t(x)$ for some $t$ which is a contradiction. Hence, $M \cap g a p(d)=\emptyset$. 
Observe that if $c \in \operatorname{gap}(d)$ then $c \in M(p / d)$, and if $c \in M$ then $c \in M(p / d)$. So, it remains to show that $M(p / d) \subset M \cup \operatorname{gap}(d)$. Let $c \in M(p / d)$. We claim that $c \in M$ or $c \in \operatorname{gap}(d)$.

Since $c \in M(p / d)$ it follows that there is $a \in M$ and $\eta \in \mathcal{L}_{A}$ so that $c=$ $\min (x) \eta(x, a, d)$. We can without loss of generality assume $M(p / d) \models \forall v, z \exists ! x \eta(x, z, v)$. Since $p(v)$ was assumed to be strongly definable, we can find a formula $\theta(v, w) \in p(v)$ with $\theta \in \mathcal{L}_{A}$ and $b \in M$ with the property

$$
M \models \forall x, z\left(\forall^{\infty} v(\theta(v, w) \rightarrow \eta(x, z, v)) \vee \forall^{\infty} v(\theta(v, w) \rightarrow \neg \eta(x, z, v))\right)
$$

We proceed by considering each case. First, we assume that $M=\forall x \forall^{\infty} v(\theta(v, w) \rightarrow$ $\neg \eta(x, z, v))$. Suppose $c \notin M$. By Lemmas 2.2.2, 2.2.3, and 2.2.4 it follows that $c>M$. Since $c \in M(p)$ and $c>M$ we have that $M(p)$ is an end extension.

Define $t(c)=\max \{\max (v)(\theta(v, w) \wedge \eta(c, z, v)): w, z \leq c\}$. Notice that this definition makes sense given that $a, b \in M$ and thus $a, b<d$. Also, $M \models \eta(c, a, d)$ and $\theta(v, w) \in p(v)$ which implies that $\theta(v, w) \wedge \eta(c, z, v)$ is true in $M$ for any $w, z \leq c$. Notice that we are considering the maximum $v$ such that $\theta(v, w) \wedge \eta(c, z, v)$ holds for all $w, z \leq c$, and $d$ is a valid input for $\mathrm{v}$. So, it follows that $t(c) \geq d$. Now define $s(d)=\max \{\min (x) \eta(x, z, d): z \leq d\}$. Since $a<d$ and $c=\min (x) \eta(x, a, d)$, it follows that $s(d) \geq c$. So, we have that $c \in \operatorname{gap}(d)$ by Fact 2.2.2.

Now assume that $M \models \exists x \exists^{\infty} v(\theta(v, w) \wedge \eta(x, z, v))$. Let $a_{0} \in M$ be given from the previous sentence. Then, $M \models \forall^{\infty} v\left(\theta(v, w) \rightarrow \eta\left(a_{0}, a, v\right)\right.$. So for a fixed $v_{0}$, $M \models \forall v\left(\theta(v, b) \wedge v \geq v_{0} \rightarrow \eta\left(a_{0}, a, v\right)\right)$. So, $M(p) \models \forall v\left(\theta(v, w) \wedge v \geq v_{0} \rightarrow \eta\left(a_{0}, a, v\right)\right)$. We know by assumption that $\theta(v, w) \in p(v)$, and $d \in M(p) \backslash M$. Then, it follows that 
since $d>v_{o}, M(p) \models \theta(d, w) \in p(d)$. Now, we have that $M(p) \models \eta\left(a_{0}, a, d\right)$. Finally, since $M(p) \models \forall v, z \exists ! x \eta(x, z, v)$, it follows that $a_{0}=c \in M$ as they both witness $\eta$

\subsection{Extensions of Models of PA along linear orders}

In this section we will show how to build an extension of a model of PA along a fixed linear order. The resulting model is similar to the model obtained in Proposition 2.2.1 however the model contains gaps for every element in our linear order sitting above the ground model. This extension was also created by Gaifman. In Proposition 2.2.1, we generated the extension using unbounded strongly definable complete $M$-types. We will now use what are known as minimal types to construct our new extension.

Definition 2.3.1. Let $M \models P A$. A complete $M$-type $p$ is rare, if for any elements $a, b \in K$ realizing $p$ such that $K$ is an extension of $M$, then the elements $a, b \in K$ are in different gaps.

Definition 2.3.2. A type $p(v)$ is minimal in a model $M$ of $P A$, if $p(v)$ is unbounded, strongly definable and rare.

Fact 2.3.1. In any model $M$ of Peano arithmetic, there is an unbounded strongly, definable, rare, complete $M$ - type.

Definition 2.3.3. Let $p(v)$ be a type of a linearly ordered model $M$. Then $p(v)$ is indiscernible whenever $c_{0}, \ldots, c_{n}, d_{0}, \ldots, d_{n} \in K \succcurlyeq M$ which realize the type $p(v)$ such that $c_{0}<\ldots<c_{n}$ and $d_{0}<\ldots<d_{n}$ then $t p_{K}\left(c_{0}, \ldots, c_{n}\right)=t p_{K}\left(d_{0}, \ldots, d_{n}\right)$ 
It turns out that we can characterize the notion of minimal type as being unbounded and indiscernible.

Lemma 2.3.1. Given a model $M$ of $P A$, if each $M$-type $p$ is complete and minimal, then $p$ is also indiscernible and unbounded.

The following lemma shows us that indiscernible types can decide formulas that are definable in a model of PA. This will be important in our main result, as we will use indiscernible types to generate an elementary extention of a given model of PA.

Lemma 2.3.2. Let $\mathcal{L}=\{<\}$ and $M$ be a model linearly ordered by $<$. If $p(v)$ is an indiscernible $M$ - type then for every $\varphi\left(v_{0}, \ldots, v_{n}\right) \in \mathcal{L}(M)$, we can find a formula $\theta(v) \in p(v)$ such that

$$
M \models \forall \bar{v}\left(\bigwedge_{i \leq n} \theta\left(v_{i}\right) \wedge \bigwedge_{i \leq n} v_{i}<v_{i+1} \rightarrow \varphi(\bar{v})\right) \vee\left(\forall \bar{v} \bigwedge_{i \leq n} \theta\left(v_{i}\right) \wedge \bigwedge_{i \leq n} v_{i}<v_{i+1} \rightarrow \neg \varphi(\bar{v})\right)
$$

We now state and prove the main theorem of this section. The result was initially proved by Gaifman and provides a way to construct an end extension of a model of PA along an arbitrary linear order [8]. We will refer to these extensions from now on as cannonical I-models, and they will be denoted $M(I)$.

Theorem 2.3.1. $M \models P A$ and a minimal complete $M-$ type $p(v)$. Let $(I,<)$ be a linear order; then there is an extension $K \succcurlyeq M$ such that $K=M \cup \bigcup_{i \in I} \operatorname{gap}_{K}\left(d_{i}\right)$. 
Proof. First we need to find the extension $K$ of $M$. Let $T=\operatorname{Diag}_{e l}(M) \cup \bigcup_{i \in I} p\left(d_{i}\right) \cup$ $\left\{d_{i}<d_{j}: i, j \in I \wedge i<j\right\}$ be a theory where each $d_{i}$ is a new constant symbol. Since $p(v)$ is a minimal complete $M$-type, we have by Lemma 2.3.1 and Lemma 2.3.2 that $T$ is a complete theory. We take $K$ to be the $\mathcal{L}_{A}$ reduct of the prime model of $T$. So, it follows that $K \succcurlyeq M$ since $p(v) \supset \operatorname{Diag}_{e l}(M)$.

We now aim to show that $K=M \cup \bigcup_{i \in I} \operatorname{gap}_{K}\left(d_{i}\right)$. Note that for each $i \in I$, $\operatorname{gap}_{K}\left(d_{i}\right)$ is defined with respect to the extension $K$. Hence, $M \cup \bigcup_{i \in I} \operatorname{gap}_{K}\left(d_{i}\right) \subseteq K$.

Now we will show that $K \subseteq M \cup \bigcup_{i \in I} \operatorname{gap}_{K}\left(d_{i}\right)$. Let $c \in K$. We must show that $c \in M \cup \bigcup_{i \in I} \operatorname{gap}_{K}\left(d_{i}\right)$. Since $c \in K$ and $K$ is the prime model of $M$, we can find $\eta \in$ $\mathcal{L}_{A}(M)$ and $d_{0}, \ldots, d_{n} \in D$ with $c=\min (x)(\eta(x, \bar{d}))$. Let $K_{0}=c l_{K}\left(M \cup\left\{d_{i}: i \leq n\right\}\right)$ be the Skolem closure or set of elements which are definable with parameters from $M \cup\left\{d_{i}: i \leq n\right\}$. Since $p$ is a rare type, and $\operatorname{gap}_{K_{0}}\left(d_{i}\right) \cap g a p_{K_{0}}\left(d_{j}\right)=\emptyset$, it follows that $\operatorname{gap}_{K_{0}}\left(d_{1}\right)<\ldots<\operatorname{gap}_{K_{0}}\left(d_{n}\right)$. Since strongly definable types are preserved in end extensions, it follows that each $d_{i}$ realizes an unbounded strongly definable type over $c l_{K_{0}}\left(M \cup\left\{d_{j}: j<i\right\}\right)$ for all $i \leq n$. Now, we proceed by applying Proposition 2.2.1 $n+1$ many times to obtain $K_{0}=M \cup \bigcup_{i \leq n} \operatorname{gap}_{K_{0}}\left(d_{i}\right)$. Clearly $c \in K_{0}$ as $c$ is definable from $K_{0}$. So, we have that either $c \in M$ or $c \in \operatorname{gap}_{K_{0}}\left(d_{i}\right)$ for some $i \leq n$. Finally, since $K_{0}$ and $K$ have the same Skolem functions it follows by Fact 2.2.2 that $c \in \operatorname{gap}_{K}\left(d_{i}\right)$. Hence, $c \in M \cup \bigcup_{i \in I} \operatorname{gap}_{K}\left(d_{i}\right)$.

\subsection{Borel reducibility of linear orders to models of PA}

In this section we will show that the isomorphism relation for countable linear orders is Borel reducible to countable models of PA. To obtain the desired reduction we need to show the following: 
1. Given two isomorphic linear orders $I$ and $I^{\prime}$, the corresponding $I$ models $M(I)$ and $M\left(I^{\prime}\right)$ respectively are isomorphic.

2. Given two isomorphic I-models of PA $M(I)$ and $M\left(I^{\prime}\right)$, the corresponding linear orders $I$ and $I^{\prime}$ are isomorphic.

3. The mapping $f: I \rightarrow M(I)$ is Borel.

Theorem 2.4.1. Consider the isomorphism equivalence relation $\cong_{L O}$ on the class countable linear orders, and $\cong_{I}$ the isomorphism equivalence relation on the class of canonical I-models. Then, there is a Borel reduction $f: I \rightarrow M(I)$ where $f$ maps a given linear order to the corresponding I-model.

Proof. Suppose $I$ and $I^{\prime}$ are isomorphic linear orders. Let $M(I)$ and $M\left(I^{\prime}\right)$ be the corresponding $I$-models. We need to show that $M(I) \cong M\left(I^{\prime}\right)$.

By Lemma 2.1.1, we know that since $I \cong I^{\prime}$, we can extend this isomorphism to an isomorphism between the Skolem hull of $I$ and $I^{\prime}$. Hence, we obtain the desired isomorphism $M(I) \cong M\left(I^{\prime}\right)$.

Consider the canonical $I$-models $M(I) \cong M\left(I^{\prime}\right)$ generated along the linear orders $I$ and $I^{\prime}$ respectively. We must show that $I \cong I^{\prime}$. By Theorem 2.3.1, we know that $M(I)=M \cup \bigcup_{i \in I} g a p\left(d_{i}\right) \cong M \cup \bigcup_{i \in I} g a p\left(d_{i}^{\prime}\right)$. Since $M(I)$ is generated by a rare type, we know that there is only one witness $d_{i}$ of the type $p$ per gap. Thus, $M(I)$ has gaps of order type $1+I$. By similar reasoning, $M\left(I^{\prime}\right)$ has gaps of order type 
$1+I^{\prime}$. Furthermore, our isomorphism $M(I) \cong M\left(I^{\prime}\right)$ maps gaps of $M(I)$ to gaps of $M\left(I^{\prime}\right)$. Hence, we obtain the induced isomorphism from $I$ to $I^{\prime}$.

Finally, we will show that the map is Borel. We note that the model $M(I)$ is the prime model of a countable model of the theory $T=\operatorname{Diag}_{e l}(M) \cup \bigcup_{i \in I} p\left(d_{i}\right) \cup$ $\left\{d_{i}<d_{j}: i, j \in I \wedge i<j\right\}$. The type of construction used to obtain $M(I)$ is a Henkin construction followed by a Skolem closure. It is a well known fact that these constructions are both Borel. Thus, we conclude that the map $f: I \rightarrow M(I)$ is Borel. 


\section{Chapter 3}

\section{BOREL COMPLEXITY OF COUNTABLE MODELS OF ZFC}

In this chapter we will adapt the arguments from Chapter 1 to show that the class of countable models of ZFGC is Borel complete. A model of ZFGC is a model that satisfies the axioms of ZF and has a well ordering of its universe. Recall that every model of PA supports built in Skolem functions. This fact was used implicitly in the construction of the canonical $I$-models from Chapter 1 . Thus, in order to adapt the construction of cannonical $I$-models in a model of set theory, we will initially look at models with global choice as they contain built in Skolem functions. It is not true, in general, that any model of set theory possesses built in Skolem functions. We also show how to modify this argument to show that countable models of any completion $T$ of $\mathrm{ZFC}$ are Borel complete.

\subsection{Types with parameters from $\omega$}

In this section, we will redefine the types seen in Chapter 1 within models of ZFGC. The following types $p(v)$ will be defined using parameters from $M$, where $M$ is a fixed model of $Z F G C$. Additionally, all of the types in this chapter will contain the formula $v<\omega^{M}$. We will refer to these types as $\omega^{M}$-types. Defining types in this 
way will allow us to obtain minimal types in models of ZFGC.

Definition 3.1.1. Let $M \models Z F G C$. Then, we say that the $\omega^{M}$-type $p(v)$ is unbounded, if $p(v) \supset\left\{\alpha \in v: \alpha \in \omega^{M}\right\}$

Definition 3.1.2. Let $M \models Z F G C$. Fix a formula $\theta \in \mathcal{L}$. We write $\exists^{\infty}(v) \theta(v)$ to abbreviate $\forall \alpha \in \omega^{M} \exists \beta \in \omega^{M}$ such that $\alpha \in \beta$ and $M \models \theta(\beta)$.

Similarly, we denote $\forall^{\infty} v \theta(v)$ to abbreviate $\exists \alpha \in \omega^{M} \forall \beta \in \omega^{M}$ such that $\alpha \in \beta$, $M \models \theta(\beta)$.

Definition 3.1.3. Let $M \models Z F G C$. Then a complete $\omega^{M}$-type $p(v)$ is strongly definable if for every formula $\varphi(v, z) \in \mathcal{L}_{M}$, we can find a formula $\theta(v) \in p(v)$ with the following property,

$$
M \models \forall z\left(\forall^{\infty} v(\theta(v) \rightarrow \varphi(v, z)) \vee \forall^{\infty} v(\theta(v) \rightarrow \neg \varphi(v, z))\right)
$$

Definition 3.1.4. Let $M \models Z F G C$ and $p(v)$ be a complete $\omega^{M}$-type. Then, $p(v)$ is definable if for any $\varphi(v, z) \in \mathcal{L}_{M}$ the set $\{z \in M: \varphi(v, z) \in p(v)\} \in \operatorname{Def}(M)$.

By Fact 2.2.1 we know that definable types exist in models of PA. In this section, we will show that definable types exist in models of ZFGC.

Definition 3.1.5. Let $M \models T$ such that $T$ is a completion of $Z F G C$. A type $p(v)$ is definable if for any formula $\varphi(u, v) \in p(v)$ we can find a formula $\sigma_{\varphi}(u)$ such that given any constant Skolem term $t$, 


$$
\varphi(y, v) \in p(v) \Leftrightarrow T \vdash \sigma_{\varphi}(t)
$$

There is a natural mapping $\varphi \mapsto \sigma_{\varphi}$ which we will call the defining scheme for the type $p(v)$.

Lemma 3.1.1. Let $M \models T$ such that $T$ is a completion of $Z F G C$. If $p(v)$ is a complete definable type containing the formula $v<\omega^{M}$ and is consistent with $T$, then $p(v)$ can be extended to a complete definable $\omega^{M}$-type $q(v) \supset p(v)$ that is consistent with $M$.

Proof. Since $p(v)$ is definable, we can fix a defining scheme $\varphi \mapsto \sigma_{\varphi}$ for $p(v)$. Define $q(v)=\left\{\varphi(b, v): b \in M \wedge M \models \sigma_{\varphi}(b)\right\}$. Clearly, $q(v) \supset p(v)$ and by construction $q(v)$ is definable. Furthermore, since $T$ is a complete theory and $q(v) \subset T$, we have that $q(v)$ is complete. We need to show that $q(v)$ is consistent.

Now we will show that the type $q(v)$ is consistent. In particular, we will show that $q(v)$ is finitely satisfiable and thus consistent by the compactness theorem. Let $\varphi\left(a_{0}, v\right), \varphi\left(a_{1}, v\right), \ldots, \varphi\left(a_{n-1}, v\right) \in q(v)$. We know that $p(v)$ is consistent with $T$, and thus by the compactness theorem is finitely realized by $T$. So, $T \vdash \forall x_{0}, \ldots, x_{n-1}\left[\bigwedge_{i<n} \sigma_{\varphi_{i}}\left(x_{i}\right) \rightarrow\right.$ $\left.\exists v \bigwedge_{i<n} \varphi_{i}\left(x_{i}, v\right)\right]$. Since $p(v)$ is definable and finitely realized by $T$, it follows that $M \models \bigwedge_{i<n} \sigma_{\varphi_{i}}\left(a_{i}\right)$. Finally, as $M \models T$, we can find an element $b \in M$ such that $M \models \bigwedge_{i<n} \varphi_{i}\left(x_{i}, b\right)$. Hence, by compactness $q(v)$ is consistent with $M$.

Lemma 3.1.2. Let $M \models Z F G C$ and $D$ be a definable set with parameters in $M$. If $\varphi(u, v)$ is a formula in the language of set theory, then we can find an unbounded definable subset $E \subseteq D$ such that $M \models \forall x \in \omega^{M}\left[\exists y \in \omega^{M} \forall z \in \omega^{M}(y \in z)(z \in E \rightarrow\right.$ $\left.\varphi(x, z)) \vee \exists y \in \omega^{M} \forall z \in \omega^{M}(y \in z)(z \in E \rightarrow \neg \varphi(x, z))\right]$ 
Proof. See the proof of Lemma 2.2.4 [5].

Remark 3.1.1. Lemma 2.2.4. of [5] is proved in the context of PA. The same argument holds in models of ZFGC since the proof relies on arithmetized Ramsey's theorem, that is, Ramsey's theorem for definable sets. The proof of Ramsey's theorem shows that the lemma holds within the context of set theory.

Theorem 3.1.1. Let $M \models T$ such that $T$ is a completion of $Z F G C$. Then, $T$ has a definable type.

Proof. First we begin by fixing an enumeration $\left\langle\varphi_{n}(u, v): n<\omega\right\rangle$ of every formula in our language. We will work in $M$, the prime model of $T$. Let $M=X_{0}$, with $D=M$ as in the Lemma 3.1.2 and $\varphi=\varphi_{0}$. By induction, assume that for a given $n, X_{n}$ is unbounded and definable. By Lemma 3.1.2 there is a an unbounded definable set $X_{n+1}$ such that $X_{n+1} \subseteq X_{n}$ with $\varphi=\varphi_{n+1}$. Furthermore, for each $n$ we can find a formula $\sigma_{n}(u)=\forall x \in M\left[\exists y \in M \forall z(y \in z)(z \in E \rightarrow \varphi(x, z))\right.$. In other words, $\sigma_{n}$

decides the parameters such that $X_{n}$ is a subset of a set defined by $\varphi_{n}(v, u)$. Thus, we let $p(v)=\left\{\varphi_{n}(u, v): b \in M \wedge M \models \sigma_{n}(b)\right\}$ be our definable type.

Corollary 3.1.1. Let $M \models Z F G C$. Then, $M$ has a definable type.

This result follows by letting $T=T h(M)$ in Theorem 3.1.1 and Lemma 3.1.1.

\subsection{End extensions of models of ZFGC generated by types}

In this section we will define the notion of a gap within a model of $Z F G C$. The gaps will be defined on the ordinals only. We will then prove the analogue of Proposition 2.2.1 concentrating only on the ordinals. 
Remark 3.2.1. Let $M \models Z F G C$. Then, $M(p)$ will refer to the prime model of the extension of $M$ generated by a complete $\omega^{M}$-type $p$. In particular, $M(p)$ is the prime model of $\operatorname{Diag}_{e l}(M) \cup p(v)$.

Now we will redefine the notion of a gap within a model of $Z F G C$. We proceed by defining gaps along $\omega^{M}$ where $M$ is our fixed model of $Z F G C$.

Definition 3.2.1. Let $M \models Z F G C$. Then, we define the following:

1. $M_{\omega}(\beta)=\left\{\alpha \in \omega^{M}:\right.$ for some Skolem function $\left.t, t(\beta) \in \omega^{M} \wedge \alpha<t(\beta)\right\}$.

2. $M_{\omega}[\beta]=\left\{\alpha \in \omega^{M}\right.$ : for any Skolem function $t$, if $t(\beta) \in \omega^{M}$ then $\left.t(\alpha)<\beta\right\}$.

3. We define the $\omega^{M}$-gap as $\operatorname{gap}_{\omega}(\beta)=M_{\omega}(\beta) \backslash M_{\omega}[d]$.

The rest of this section will be devoted to proving the analogue of Theorem 2.2.1 in $Z F G C$. We will prove this theorem by only considering the $\omega$ of our fixed model $M$ of ZFGC and its extension $M(p)$. First we recall the following fact about gaps.

Fact 3.2.1. Let $\alpha, \beta \in \omega^{M}$ such that $M \models Z F G C$. Then, $\alpha \in \operatorname{gap}(\beta)$ if and only if there are Skolem functions $f, g$ such that $\beta \leq f(\alpha)$ and $\alpha \leq g(\beta)$.

Remark 3.2.2. By the same reasoning as stated in Remark 2.2.3, we recall that gaps in models of ZFGC are also disjoint.

Definition 3.2.2. Let $M \models Z F G C$. Then, we say that $K$ is a conservative extension of $\omega^{M}$ if $M \subset K$ such that for any definable set $X \in \operatorname{Def}(K)$ such that $X \subset \omega^{K}, X \cap M \in D E F(M)$. 
Lemma 3.2.1. Let $M \models Z F G C$ and $p(v)$ be a complete $\omega^{M}$-type. If $p$ is a definable type, then the $\omega^{M(p)}$ is a conservative extension of $M$.

Proof. Let $p$ be a definable $\omega^{M}$-type, and $\beta \in M(p)$ realizing $p$. Fix a definable set $X \in \operatorname{Def}(M(p))$. Then, $X=\{\gamma \in M(p): M(p) \models \theta(\alpha, \gamma)\}$ such that $\theta$ is a formula in the language of set theory and $\alpha \in M(p)$. Since $\alpha \in M(p), \alpha=\eta(\beta)$ for some formula $\eta$ in the language of set theory. We complete the proof by calculating $X \cap M$,

$$
\begin{aligned}
X \cap M & =\{\delta \in M: M(p) \models \theta(\alpha, \gamma)\} \\
& =\{\delta \in M: M(p) \models \theta(\eta(\beta), \gamma)\} \\
& =\{\delta \in M: \theta(\eta(\beta), \gamma) \in p\} .
\end{aligned}
$$

Finally, since $p$ is definable, it follows that $X \cap M \in \operatorname{Def}(M)$ and thus $M(p)$ is a conservative extension.

Definition 3.2.3. Let $M \models Z F G C$. We say that $K$ is an $\omega^{M}$ end extension of $M$ if for any $\alpha \in \omega^{K} \backslash \omega^{M}, \beta \in \alpha$ for any ordinal $\beta \in \omega^{M}$.

Lemma 3.2.2. Let $M \models Z F G C$ and $K \supset \omega^{M}$ be a conservative extension of $K$. Then, $K$ is an $\omega^{M}$ end extension of $\omega^{M}$.

Proof. Let $\alpha \in \omega^{K} \backslash \omega^{M}$. Consider the set $X=\left\{\beta \in \omega^{M}: \beta \in \alpha\right\}$. Since $K$ is conservative and $X$ is definable, $X \cap M \in \operatorname{Def}(M)$. We claim that $X \cap \omega^{M}=\{\beta \in$ $\left.\omega^{M}: \beta \in \alpha\right\}=\omega^{M}$.

We proceed by induction on $\alpha$. Clearly, if $\gamma=\emptyset$, then $\gamma \in X \cap \omega^{M}$. If $\gamma \in \omega^{M}$, then $\gamma+1 \in \omega^{M}$. Hence, $\gamma \in X \cap \omega^{M}$ since $\alpha \in \omega^{K} \backslash \omega^{M}$. Hence, $K$ is an $\omega^{M}$ end extension. 
Now we are ready to state the analogue of Proposition 2.2.1 within a model of $Z F G C$.

Proposition 3.2.1. Let $M \models Z F G C$, and $p$ be a definable $\omega^{M}$-type. Then, $\omega^{M(p)}=$ $\omega^{M} \cup \operatorname{gap}_{\omega}(d)$.

The proof of Proposition 3.2.1 follows using the same argument as in Proposition 2.2.1. One simply replaces end extension with $\omega^{M}$ end extension etc.

\subsection{Canonical extensions of models of ZFGC along linear orders}

In this section we will build an extension of the ordinals of a fixed model of $Z F G C$ along a linear order of ordinals. We will begin by defining minimal types with ordinal parameters. Similar to the construction in Chapter 1, adjoining a minimal type will allow us to construct an extension along a linear order in such a way that all of the gaps above the ground model are disjoint.

Definition 3.3.1. Let $M \models Z F G C$. Then, an $\omega^{M}$-type $p(\alpha)$ is rare, if for any ordinals $\alpha, \beta \in K$ such that $K$ is an $\omega^{M}$-end extension of $\omega^{M}$, then $\alpha$ and $\beta$ are in unique gaps.

Definition 3.3.2. Let $M \models Z F G C$. Then, $p(\beta)$ is minimal with respect to $\omega^{M}$ if $p(\beta)$ is rare, strongly definable and unbounded. 
As shown in the previous section, we know that definable types in models of $Z F G C$ exist. Minimal types as will be shown exist in models $Z F G C$.

Proposition 3.3.1. Let $N \models Z F G C$ and $T$ be a completion of $Z F G C$. If $p$ is a minimal type, then $p$ is definable.

Proof. Suppose $p$ is not definable. Let $\varphi(u, x)$ witness that $p$ is not a definable type. Consider the set of formulas

$$
\begin{aligned}
\psi(\alpha, \beta, \gamma) & =p(\beta) \cup(\gamma) \cup\left\{s<x: \text { s is a constant Skolem term } \wedge s \in \omega^{M}\right\} \\
& \cup\left\{t(x)<y<z: t(x) \text { is a Skolem term } \wedge t(x) \in \omega^{M}\right\} \\
& \cup\{\forall q \in x[\varphi(q, y) \leftrightarrow \theta(q, z)]\} \cup\{\varphi(x, y) \leftrightarrow \neg \varphi(x, z)\} .
\end{aligned}
$$

We claim that $\psi(\alpha, \beta, \gamma)$ is consistent with $T$.

If we take any finite subset $X \subseteq \psi(x, y, z)$, it is clear that $X \models \psi(x, y, z)$. Hence, by the compactness theorem $\psi(\alpha, \beta, \gamma)$ is consistent with $T$. Let $M \models T$ such that $a, b, c \in \omega^{M}$ witness $\psi(a, b, c) \models T$. Fix a prime model $M^{\prime}$ of $T$. Consider $\omega^{M^{\prime}}$, the $\omega$ of the prime model $M^{\prime}$. Notice that $\omega^{M^{\prime}}<a$ since $a$ satisfies $s<x$ for any constant Skolem term $s$. Also, $\omega^{M^{\prime}}<a<\operatorname{gap}(b)$ since $t(a)<b<c$ for any Skolem function $t$. Thus, $a \notin M_{\omega}^{\prime}(b)$. Since $p$ is minimal, we know that $p$ is a rare type. So, $b<g a p(c)$ since the gaps of $b$ and $c$ are disjoint. Furthermore, $c \notin M_{\omega}(a, b)$. Thus, it follows that $M_{\omega}(b) \prec M_{\omega}(a, b) \prec M_{\omega}(a, b, c)$. Finally, since $c \notin M_{\omega}(a, b)$ and $b, c$ witness $p$, we have $M_{\omega}(a, b, c)=M_{\omega}(b, c)$. Hence, $M_{\omega}(b)$ is not a minimal extension of $M_{\omega}$ which is a contradiction.

Definition 3.3.3. Let $M \models Z F G C$ and $p(\beta)$ be a type with parameters in $\omega^{M}$. We 
say that $p(\beta)$ is $\omega^{M}$ indiscernible if there are increasing sequences $\alpha_{0}, \ldots, \alpha_{n}, \beta_{0}, \ldots, \beta_{n} \in$ $K$ with respect to $\epsilon$ witnessing $p(\beta)$ such that $K$ is an $\omega^{M}$-end extension of $M_{\omega}$ then $p\left(\alpha_{0}, \ldots, \alpha_{n}\right)=p\left(\beta_{0}, \ldots, \beta_{n}\right)$.

As in Chapter 1, we will provide a characterization of minimal types with ordinal parameters.

Lemma 3.3.1. Let $M \models Z F G C$ and $p(\gamma)$ be a minimal complete $\omega^{M}-$ type. Then, $p(\gamma)$ is ordinal indiscernible.

We restate Lemma 2.3.2 from the first chapter as it will be used to prove the following theorem. Lemma 2.3.2 will be applied to the ordinals of a fixed model $M \models Z F G C$.

Lemma 3.3.2. Let $\mathcal{L}=\{<\}$ and $M$ be a model linearly ordered by $<$. If $p(v)$ is an indiscernible $M$ - type then for every $\varphi\left(v_{0}, \ldots, v_{n}\right) \in \mathcal{L}(M)$, we can find a formula $\theta(v) \in p(v)$ such that

$$
M \models \forall \bar{v}\left(\bigwedge_{i \leq n} \theta\left(v_{i}\right) \wedge \bigwedge_{i \leq n} v_{i}<v_{i+1} \rightarrow \varphi(\bar{v})\right) \vee \forall \bar{v}\left(\bigwedge_{i \leq n} \theta\left(v_{i}\right) \wedge \bigwedge_{i \leq n} v_{i}<v_{i+1} \rightarrow \neg \varphi(\bar{v})\right) .
$$

Theorem 3.3.1. Let $M \models Z F G C$ and $p(\gamma)$ be an $\omega^{M}$-minimal complete $M-$ type and $(I,<)$ a linearly ordered set. Then, there is an $\omega^{M}$-end extension $\omega^{M} \prec K_{\omega}$ such that $X=\left\{\alpha_{i}: i \in I\right\} \subset K$ and $K_{\omega}=M_{\omega} \cup \bigcup_{i \in I} \operatorname{gap}_{\omega}\left(\alpha_{i}\right)$.

Proof. We first construct the ordinal end extension $K$. Let $T=\operatorname{Diag}_{e l}\left(\omega^{M}\right) \cup$ $\bigcup_{i \in I} p\left(\alpha_{i}\right) \cup\left\{\alpha_{i} \in \alpha_{j}: i<j \wedge i, j \in I\right\}$ such that each $\alpha_{i}$ is a new constant symbol. Now let $K$ be the prime model pf the theory $T$. Hence, $K$ is an $\omega^{M}$-end extension of $K$. It remains to show that $K=\omega^{M} \cup \bigcup_{i \in I} \operatorname{gap}_{\omega}\left(\alpha_{i}\right)$. As in Theorem 2.2.1 we know that $\omega^{M} \cup \bigcup_{i \in I} g a p_{\omega}\left(\alpha_{i}\right) \subset K$. We proceed by showing that $K \subset \omega^{M} \cup \bigcup_{i \in I} g a p_{\omega}\left(\alpha_{i}\right)$. 
Let $\beta \in K$. Since $K$ is a prime model, we can find a formula $\eta$ in the language of set theory and $\alpha_{0}, \alpha_{1}, \ldots, \alpha_{n} \in X$ such that $\beta \eta\left(\alpha_{0}, \alpha_{1}, \ldots, \alpha_{n}\right)$. Now, take the Skolem closure of $c_{K}\left(\omega^{M} \cup\left\{\alpha_{i}: i \leq n\right\}\right)=K_{0}$. Since $p(\gamma)$ is a rare type, the gaps of $\left\{\alpha_{i}: i \leq n\right\}$ are disjoint. So, we have that $\operatorname{gap}_{K_{0}}\left(\alpha_{1}\right)<\ldots<g a p_{K_{0}}\left(\alpha_{n}\right)$. Since $p(\gamma)$ is strongly definable, each $\alpha_{i}$ witnesses a strongly definable type. Thus, we apply Proposition 3.2.1 $n+1$ many times to obtain the structure $K_{0}=\omega^{M} \cup \bigcup_{i \leq n} g a p_{K_{0}}\left(\alpha_{i}\right)$. Now, it follows that $\beta$ is an element of $\omega^{M}$ or one of the gaps $g a p_{K_{0}}\left(\alpha_{i}\right)$ for some $i \leq n$. To finish the proof, we note that $K_{0}$ and $K$ have the same Skolem functions. So, we conclude that $\beta \in \omega^{M}$ or $\beta \in \operatorname{gap}_{K}\left(\alpha_{i}\right)$ for some $i \leq n$. Thus $K=\omega^{M} \cup \bigcup_{i \in I} \operatorname{gap}_{\omega}\left(\alpha_{i}\right)$.

\subsection{Classification of countable models of ZFGC}

In this section we will show that countable models of $Z F G C$ are Borel complete. As noted in Chapter 1, countable models of $P A$ are Borel complete. Thus, it suffices to show that there is a Borel reduction from countable models of $L O$ to countable models of $Z F G C$.

Similar to the argument of Theorem 2.4.1, we obtain the following result:

1. Given two isomorphic countable linear orders $I \cong I^{\prime}$, the corresponding $I$ models of $Z F G C M(I) \cong M\left(I^{\prime}\right)$ respectively are isomorphic.

2. Given two isomorphic countable $I$ models of $Z F G C M(I) \cong M\left(I^{\prime}\right)$, the corresponding linear orders $I$ and $I^{\prime}$ are isomorphic. 
3. The mapping $f: I \rightarrow M(I)$ is Borel.

Theorem 3.4.1. Consider the isomorphism equivalence relation $\cong_{L O}$ on the class of countable linear orders, and $\cong_{I}$ the isomorphism equivalence relation on the class of canonical I-models of completions $T$ of ZFGC. Then, assuming $T$ is consistent, there is a Borel reduction $f: I \rightarrow M(I)$ where $f$ maps a given linear order to the corresponding I-model of ZFGC. In particular, models of ZFGC are Borel complete.

We will conclude this chapter by modifying the previous result to show that countable models of ZFC are Borel complete.

Corollary 3.4.1. Let $T$ be a completion of ZFC. Assuming $T$ is satisfied by a transitive model of $Z F C$, the class of countable models of $T$ are Borel complete.

Proof. Let $T \supset Z F C$ be complete and $M \models T$ such that $M$ is transitive. Given $M \models T$, we can find a forcing extension $N$ of $M$ such that $N \models T$ and contains a choice function $F$ that is defined on all non-empty sets of $N$ (this can be found in [4]). So, we have that $N \models Z F C+G C$ such that $F$ is a global choice function defined on $N$. Without loss of generality, let $F$ be defined such that for any set $X$ in which $X \cap O R D \neq \emptyset, F(X)=$ least ordinal in $X$.

Given the model $N$ and a linear order $I$, we can carry out the construction used earlier in this chapter to obtain the canonical $I$-model $N(I)$. We now proceed to modify the Borel reduction to obtain the result. In particular, we argue that this construction does not need to make reference to the global choice assumption.

First, let $I$ and $I^{\prime}$ be countable linear orders such that $I \cong I^{\prime}$. By Theorem 3.4.1 
we can obtain an isomorphism $M(I) \cong M\left(I^{\prime}\right)$ of canonical $I$-models of ZFGC. Then, it follows that the reducts $M$ and $M^{\prime}$ of the models $M(I)$ and $M\left(I^{\prime}\right)$ to the language without the global choice function are isomorphic.

Let $M(I) \cong M\left(I^{\prime}\right)$ be canonical $I$-models of ZFGC. We claim that the gaps of $\omega^{M(I)}$ and $\omega^{M\left(I^{\prime}\right)}$ can be identified as isomorphic to $I$ and $I^{\prime}$ respectively. We identify these gaps by choosing the least ordinal that satisfies a formula rather than using the Skolem term $t$ in the definition of $\omega^{M}$-gaps.

Thus, we obtain an isomorphism between the gaps of $M(I)$ and $M\left(I^{\prime}\right)$ which are isomorphic to $I$ and $I^{\prime}$ respectively. Notice that we have established this isomorphism without making reference to the global choice function. Hence, we obtain the desired Borel reduction from countable linear orders to countable models of ZFC. 


\section{Chapter 4}

\section{THE CLASSIFICATION OF WELL-FOUNDED MODELS OF ZFC}

In the previous chapter we showed that the class of countable models of ZFC is Borel Complete. It is natural to consider the classification problem for well-founded models of set theory. Is this class Borel complete, or does this class of structures decrease in complexity? In this section, we do not claim to answer this question. On the other hand, this section will begin to generate some of the tools needed to answer this question.

In order to study the classification problem for well-founded models of set theory, we need a way to obtain continuum many non isomorphic well-founded models of some completion $T$ of ZFC. We first note that we can easily obtain continuum many non isomorphic well-founded models of ZFC, if we allow for these models to have different theories. A more interesting question is whether or not we can find continuum many non isomorphic well-founded models of a fixed completion $T$. We first consider substructures of Gödel's $L$. 


\subsection{Generating $\omega_{1}$ many non-isomorphic well-founded mod- els of a completion $T$ of $Z F C$}

Consider a fixed completion $T$ of $Z F C$. We claim that there are $\omega_{1}$-many countable models of ZFC, assuming $\omega_{1}^{V}$ is inaccessible in $L$. The next two lemmas will be stated in more generality, but will imply the previous claim. We first show that we can find at least one such substructure of $L_{\kappa}$, assuming $\kappa$ is an inaccessible cardinal.

Lemma 4.1.1. If $\kappa$ is inaccessible, then there there is a cardinal $\alpha<\kappa$ such that $L_{\alpha} \prec L_{\kappa}$.

Proof. Let $\kappa$ be inaccassible. Then, there is a model of set theory $L_{\kappa} \models Z F C$. By the Lowenheim-Skolem theorem, there is a countable substructure $N_{\alpha_{0}} \prec L_{\kappa}$. Let $X_{\alpha_{0}}$ be the Skolem hull of $N_{\alpha_{0}}$, and $L_{\alpha_{0}}$ be the smallest $L_{\gamma}$ such that $N_{\alpha_{0}} \prec L_{\alpha_{0}}$. We can continue this construction to obtain the sequence $N_{\alpha_{0}}, N_{\alpha_{1}}, \ldots, N_{\alpha_{\xi}}, \ldots$ such that $\xi<\gamma$ and $\gamma<\kappa$. Since each $N_{\alpha_{\xi}} \prec L_{\kappa}$, it follows by the elementary chain lemma that $N=\bigcup_{\xi<\gamma} N_{\alpha_{\xi}} \prec L_{\kappa}$. Notice that $N=\bigcup_{\xi<\gamma} L_{\alpha_{\xi}}$. Finally, let $\alpha=\lim _{\xi<\gamma} \alpha_{\xi}$. Then, we have that $N=L_{\alpha} \prec L_{\kappa}$.

We now claim that we can find a club sets worth of $L_{\alpha} \prec L_{\kappa}$.

Lemma 4.1.2. The set $X=\left\{\alpha<\kappa: L_{\alpha} \prec L_{\kappa}\right\}$ is club.

Proof. First we note that $X$ is unbounded by using the construction in the previous theorem. To show that $X$ is closed, we let $\alpha_{0}, \ldots \alpha_{\xi}, .$. be elements of $X$ such that $\alpha=\lim _{\xi<\gamma} \alpha_{\xi}$ where $\gamma<\kappa$. We claim that $\alpha \in X$. 
Since $\alpha_{0}, \ldots \alpha_{\xi}, .$. are elements of $X$, we know that $L_{\alpha_{\xi}} \prec L_{\kappa}$ for each $\alpha_{\xi}$. By the elementary chain lemma, it follows that $\bigcup_{\xi<\gamma} L_{\xi}=L_{\sup \left\{\alpha_{\xi}: \xi<\gamma\right\}}=L_{\alpha} \prec L_{\kappa}$. Hence, $\alpha \in X$. Thus, $X$ is closed.

Now, the fact that there are $\omega_{1}$-many countable models, provided $\omega_{1}^{V}$ is inaccessible in $L$, follows from Lemma 4.1.1 and Lemma 4.1.2. Since $L \models \omega_{1}^{V}$ is inaccessible, and $L \models \omega_{1}^{V}$ is club, we have that there are $\omega_{1}$-many models $L_{\alpha} \prec L_{\omega_{1}}$, if $\omega^{V}$ is inaccessible. Moreover, these are countable in $V$. This proves the following theorem:

Theorem 4.1.1. If $\omega_{1}^{V}$ is inaccessible in $L$, then there are $\omega_{1}$-many countable models of the theory $T=T h\left(L_{\omega_{1}}\right)$.

\subsection{Generating $2^{\omega}$ many non-isomorphic well-founded mod- els of $Z F C$}

In this section, we examine an argument provided by Enayat that allows us to construct $2^{\omega}$ non-isomorphic well-founded models of a completion $T$ of ZFC [2]. The idea is to fix a countable transitive model $M \models T$, and then use Cohen forcing to generate $2^{\omega}$ many mutually generic filters. Since the models we produce are transitive, it will then follow that any two of our generics will produce non-isomorphic models that are well-founded.

Theorem 4.2.1. Let $M \models T$ be a countable transitive model such that $Z F C \subset T$ is complete, then there are $2^{\omega}$ many well-founded non-isomorphic models of $T$. 
Proof. Let $M \models T$ and consider the Cohen forcing poset $\mathbb{P}=2^{<\omega}$ in $M$. We start by enumerating all of the open dense sets $D_{n} \subseteq \mathbb{P} \times \mathbb{P}$. Then, we recursively construct a set of conditions in our poset $\left\{p_{t}: t \in 2^{<\omega}\right\}$ such that

1. if $s \leq t$ then $p_{t} \leq p_{s}$

2. if $|s|=|t|=n$ and $s \neq t$ then $\left(p_{s}, p_{t}\right) \in D_{n}$.

In order to carry out the construction for items 1 and 2, assume that we have constructed level $n$ of our tree. That is, $p_{s}$ has been defined for any $s \in 2^{<\omega}$ such that $|s|=n$. We now construct level $n+1$, and proceed by sub-induction on $j \in$ $\left\{0, \ldots,\left(\begin{array}{c}2^{n-1} \\ 2\end{array}\right)\right\}$. If $j=0$, we let $p_{s}^{0}=p_{s \mid n}$ for any $s \in 2^{<\omega}$ such that $|s|=n+1$. Assume by induction that the $p_{s}^{j}$ have been defined for any $s \in 2^{<\omega}$ such that $|s|=n+1$. We define $p_{s}^{j+1}$ in the following way: Let $\left(t, t^{\prime}\right)$ be the $j+1$ st pair of elements of $2^{n+1}$, and let $\left(p_{t}^{j+1}, p_{t^{\prime}}^{j+1}\right) \leq\left(p_{t}^{j}, p_{t^{\prime}}^{j}\right)$ such that $\left(p_{t}^{j+1}, p_{t^{\prime}}^{j+1}\right) \in D_{n}$. Furthermore, we let $p_{s}^{j+1}=p_{s}$ whenever $s \neq t, t^{\prime}$.

Then, it follows that for any branch $x \in 2^{\omega}$ we have a generic filter $g_{x}=\left\langle\left\{p_{s}\right.\right.$ : $s \subset x\}\rangle$. By property 2, it follows that if $x, x^{\prime} \in 2^{<\omega}$ and $x \neq x^{\prime}$, then $g_{x}$ and $g_{x^{\prime}}$ are mutually generic. So, in particular $M\left[g_{x}\right] \cap M\left[g_{x^{\prime}}\right]=M$, and $M\left[g_{x}\right] \neq M\left[g_{x^{\prime}}\right]$. Finally, since forcing extensions via Cohen forcing are transitive, it follows that if $x \neq x^{\prime}$ then $M\left[g_{x}\right] ¥ M\left[g_{x^{\prime}}\right]$. Hence, it follows that there are $2^{\omega}$ many well-founded non-isomorphic models of $T$.

\subsection{Generating $E_{0}$ many non-isomorphic well-founded mod- els of a completion $T$ of $Z F C$}

In this section we will adapt our construction from section 4.2 to generate $E_{0}$ many non-isomorphic well-founded models of $Z F C$. 
Theorem 4.3.1. Let $M \models T$ be a countable transitive model such that $Z F C \subset T$ is complete, then there are $E_{0}$-many well-founded non-isomorphic models of $T$.

Proof. Let $M \models T$ and consider the Cohen forcing poset $\mathbb{P}=2^{<\omega}$ in $M$. We start by enumerating all of the open dense sets $D_{n} \subseteq \mathbb{P} \times \mathbb{P}$ in such a way that $D_{n+1} \subseteq D_{n}$. We require that $D_{n+1} \subseteq D_{n}$ so that meeting only infinitely many is equivalent to meeting them all. Then, we recursively construct a set of conditions in our poset $\left\{p_{t}: t \in 2^{<\omega}\right\}$ such that

1. if $s \leq t$ then $p_{t} \leq p_{s}$

2. if $|s|=|t|=n$ and $s \neq t$ then $\left(p_{s}, p_{t}\right) \in D_{n}$.

We proceed by adapting our construction of items 1 and 2 so that we generate $E_{0}$ many mutual generics. The set up is the same as in Theorem 4.2.1. Now, we let $\left(t, t^{\prime}\right)$ be the $j$ th pair such that $t(n)=0$ and $t^{\prime}(n)=1$. Then, we find a pair of conditions $\left(p_{t}^{j} \frown q_{t}^{j}, p_{t^{\prime}}^{j} \frown q_{t^{\prime}}^{j}\right) \leq\left(p_{t}^{j}, p_{t^{\prime}}^{j}\right)$ such that $\left(p_{t}^{j} \frown q_{t}^{j}, p_{t^{\prime}}^{j} \frown q_{t^{\prime}}^{j}\right) \in D_{n}$ and $\left|q_{t}^{j}\right|=\left|q_{t^{\prime}}^{j}\right|$. To finish the construction, we let

$$
p_{s}^{j+1}= \begin{cases}p_{s}^{j} \frown q_{t}^{j}, & \text { if } s(n)=0 \\ p_{t}^{j} \frown q_{t^{\prime}}^{j}, & \text { if } s(n)=1 .\end{cases}
$$

Thus, we obtain items 1 and 2 by induction. It then follows that given $x, x^{\prime} \in 2^{\omega}$, if $x E_{0} x^{\prime}$ then $g_{x} E_{0} g_{x^{\prime}}$ since we always add the same extension after some finite stage $n$. Thus, if we have to generics $g$ and $g^{\prime}$ such that $g E_{0} g^{\prime}$, then they generate the same model $M[g]=M\left[g^{\prime}\right]$. Furthermore, if $x$ and $x^{\prime}$ are not $E_{0}$ related, then we meet infinitely many of the open dense $D_{n}$ 's, which amounts to meeting all of them. Hence, in this case the models produced are non-isomorphic. 


\section{Bibliography}

[1] Samuel Coskey and Roman Kossak. The complexity of classification problems for models of arithmetic. Bull. Symbolic Logic, 16(3):345-358, 2010.

[2] Ali Enayat. Counting models of set theory. Fund. Math., 174(1):23-47, 2002.

[3] Harvey Friedman and Lee Stanley. A Borel reducibility theory for classes of countable structures. J. Symbolic Logic, 54(3):894-914, 1989.

[4] Thomas Jech. Set theory. Springer Monographs in Mathematics. Springer-Verlag, Berlin, 2003. The third millennium edition, revised and expanded.

[5] Roman Kossak and James H. Schmerl. The structure of models of Peano arithmetic, volume 50 of Oxford Logic Guides. The Clarendon Press, Oxford University Press, Oxford, 2006. Oxford Science Publications.

[6] David Marker. Model theory, volume 217 of Graduate Texts in Mathematics. Springer-Verlag, New York, 2002. An introduction.

[7] Tin Wok. Model theory of arithmetic, lecture 10: Strongly definable types, 10 December, 2014. http://www.logic.univie.ac.at/ wongt9/teach/modelarith/ 10_strdeftp.pdf.

[8] Tin Wok. Model theory of arithmetic, lecture 11: Minimal types, 17 December, 2014. http://www.logic.univie.ac.at/ wongt9/teach/modelarith/11_ mintp.pdf.

[9] Tin Wok. Model theory of arithmetic, lecture 9: The Mac Dowell-Specker Theorem, 3 December, 2014. http://twong.impan.pl/teach/modelarith/9_ macspeck.pdf. 